\title{
3 Research Square

\section{An asymmetric mode-localized mass sensor based on the electrostatic coupling of different structural modes with distributed electrodes}

Jiahao Song

Dalian University of Technology

Ming Lyu

Dalian University of Technology

Najib Kacem

Universite Bourgogne Franche-Comte

Jian Zhao ( $\square$ jzhao@dlut.edu.cn )

Dalian University of Technology https://orcid.org/0000-0002-2609-8259

Pengbo Liu

Dalian University of Technology

Yu Huang

Dalian Ocean University

Kefeng Fan

China Electronics Standardization Institute

\section{Research Article}

Keywords: mode-localized sensor, asymmetric mode coupling, distributed electrodes, mass sensing

Posted Date: March 31st, 2021

DOl: https://doi.org/10.21203/rs.3.rs-323305/v1

License: (9) (i) This work is licensed under a Creative Commons Attribution 4.0 International License. Read Full License 


\section{Abstract}

Mode-localization sensor with amplitude ratio as output metric has shown excellent potential in the field of micro-mass detection. In this paper, an asymmetric mode -localized mass sensor with a pair of electrostatically coupled resonators of different thickness is proposed. Partially distributed electrodes are introduced to ensure the asymmetric mode coupling of second and third order modes while actuating the thinner resonator by the distributed electrode. The analytical dynamic model is established by EulerBernoulli theory and solved by harmonic balance method (HBM) combined with asymptotic numerical method (ANM). Detailed investigations on the linear and nonlinear behavior, critical amplitude as well as the sensitivity of the sensor are performed. The sensitivity of the proposed sensor can be enhanced by about 20 times compared to first order mode-localized mass sensors. Furthermore, by exploiting the nonlinearities while driving the device beyond the critical amplitude for the in-phase mode, the sensor performs a great improvement in sensitivity up to 1.78 times. Besides, the influence of the decrease of coupling voltage is studied, which gives a good reference to avoid mode aliasing.

\section{Full Text}

This preprint is available for download as a PDF.

\section{Figures}

(a)

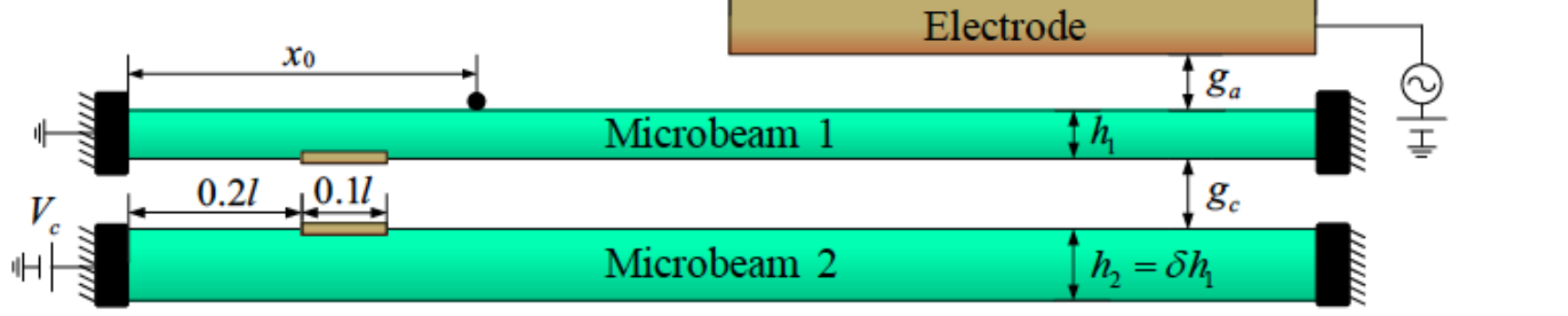

(b)

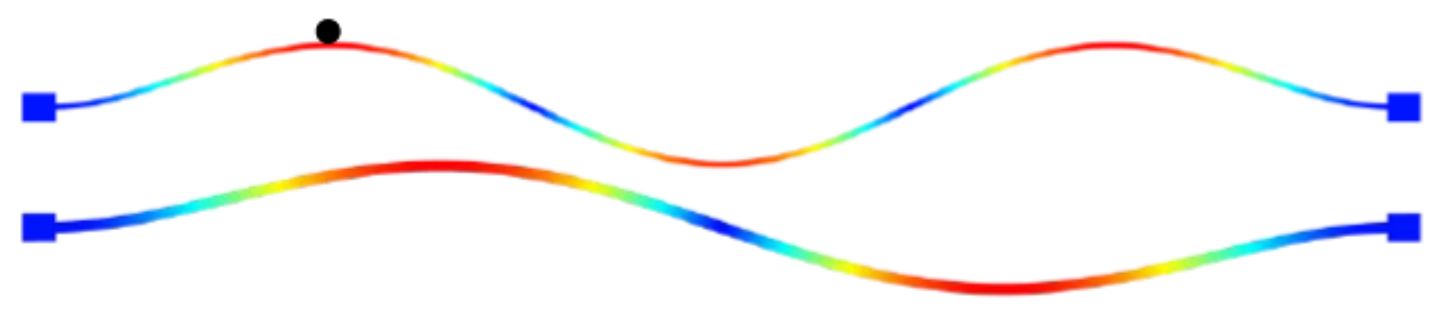

Figure 1

(a) sketch of proposed mass sensor, (b) sketch of different modes coupling 


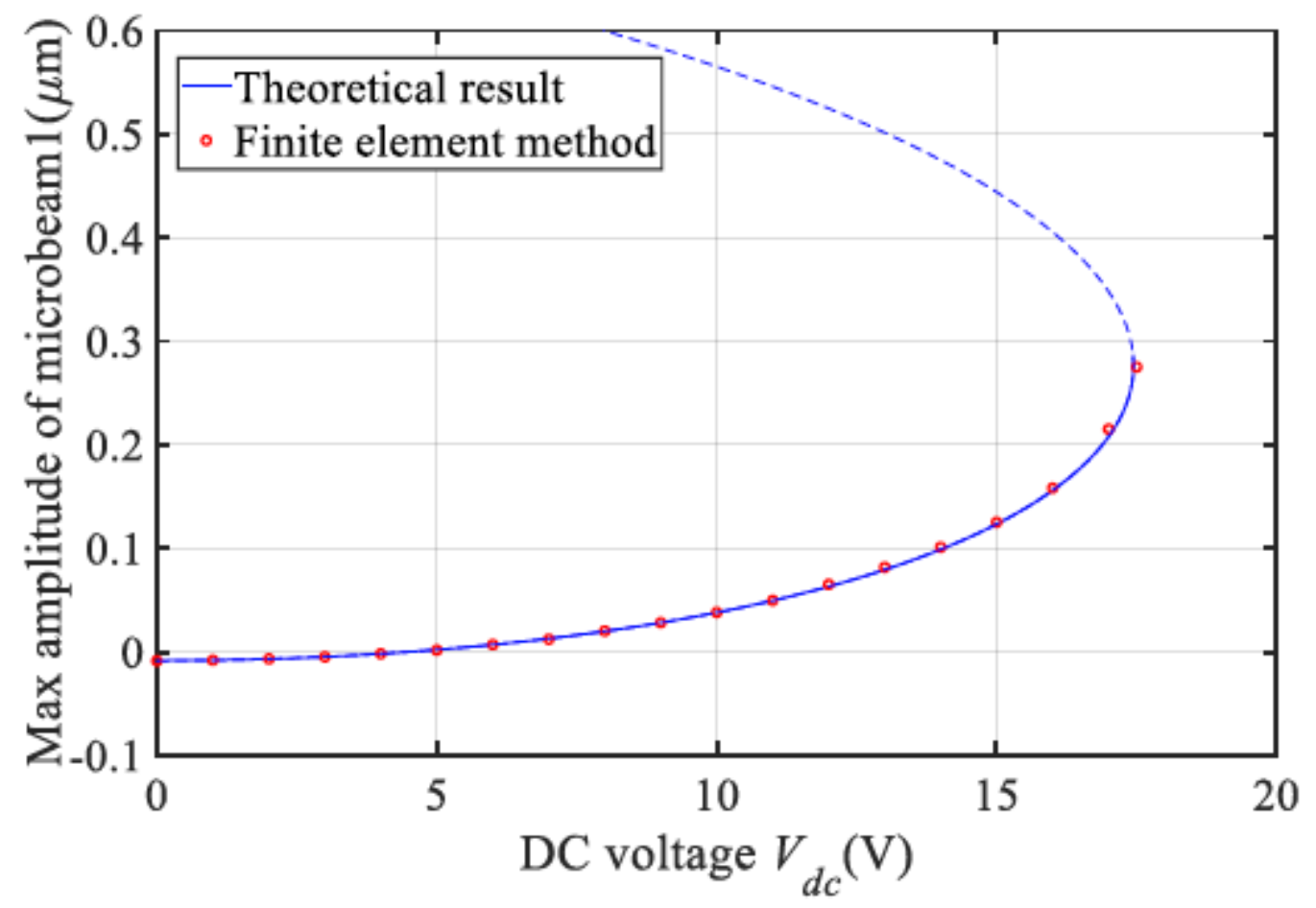

Figure 2

Electrostatic driven displacements at the middle point of microbeam 1
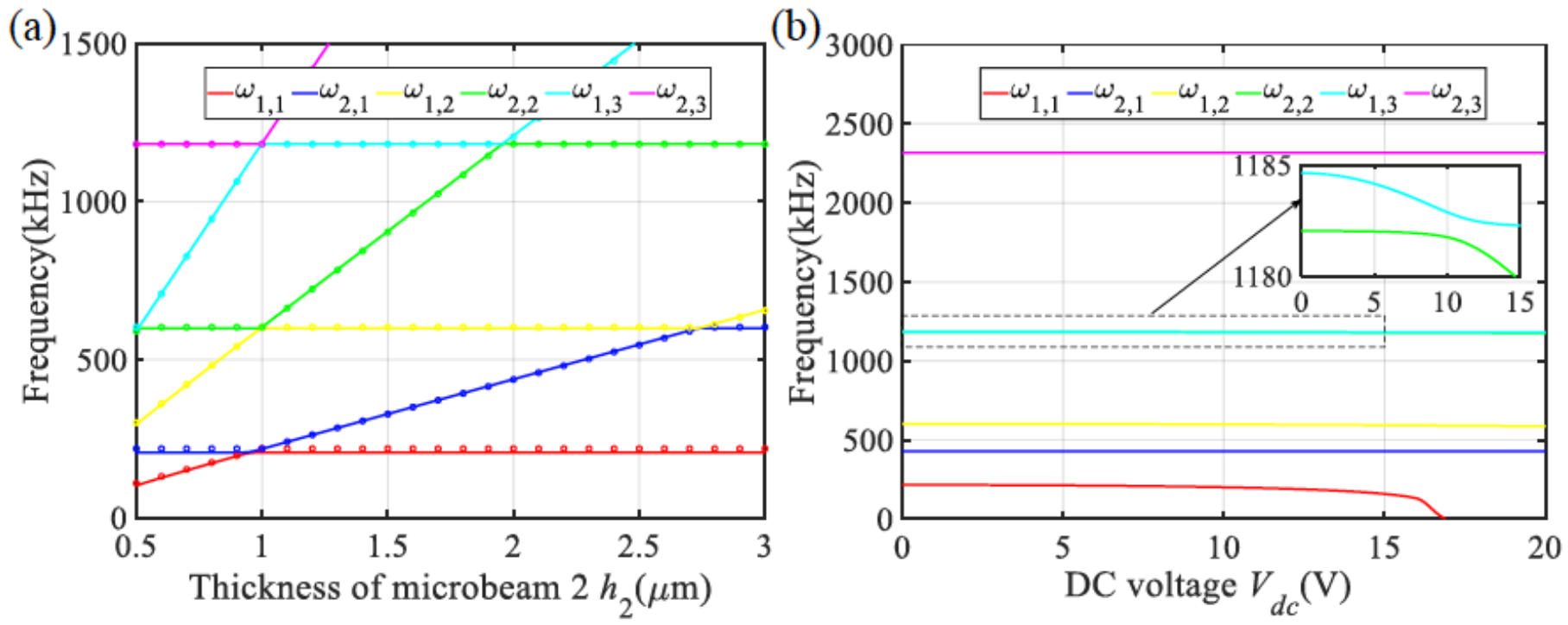

Figure 3

Eigenvalue frequencies under different thicknesses of microbeam 2 and DC voltage. (a) eigenvalue frequencies versus various of thickness of microbeam 2 with Vdc $=10 \mathrm{~V}$ (the solid lines and circles 
corresponding to theoretical results and COMSOL results, respectively), (b) eigenvalue frequencies versus various of $\mathrm{DC}$ voltage with $\mathrm{h} 2=1.956 \mu \mathrm{m}$
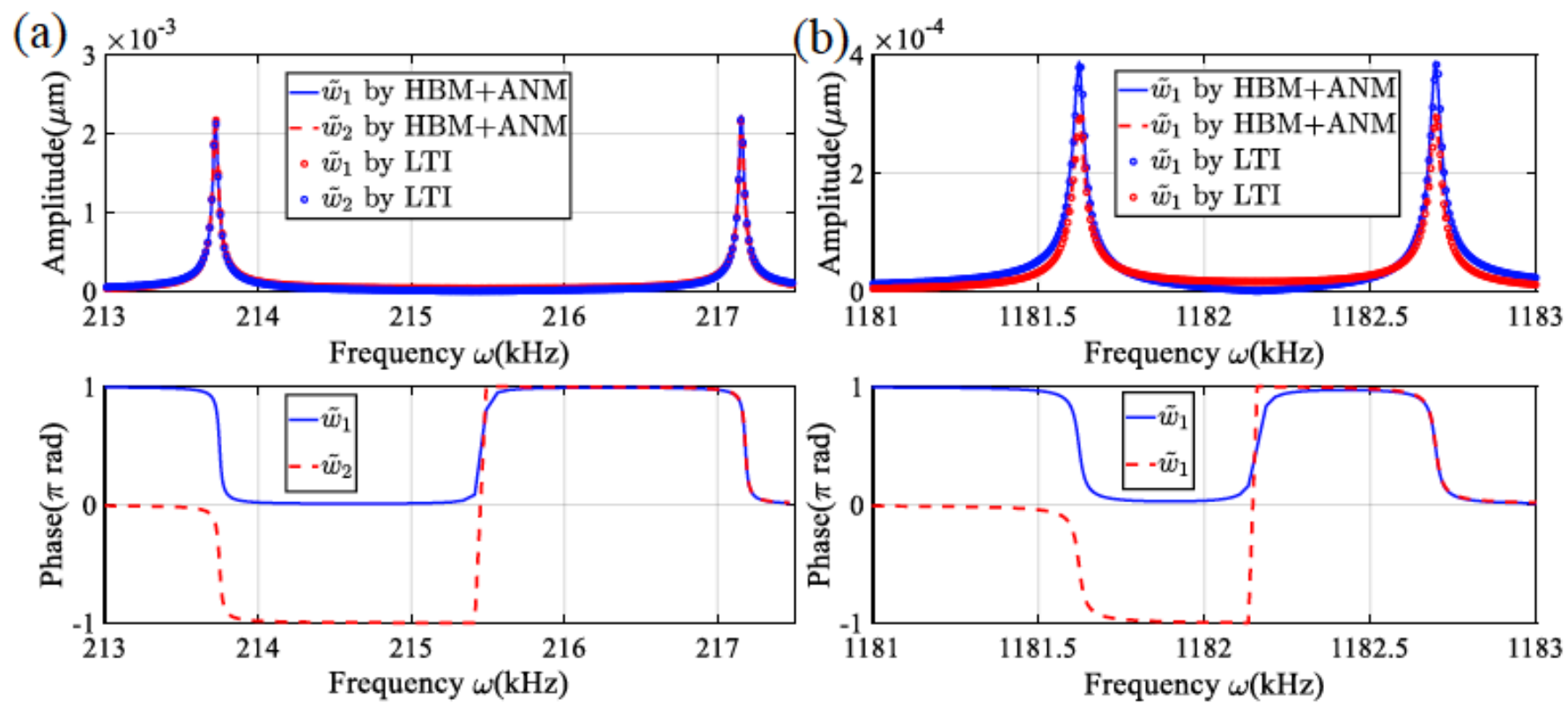

\section{Figure 4}

Linear amplitude-frequency and phase-frequency curves of the two resonators with first order coupling (a) and third-second modes coupling (b) when $V_{c}=10 \mathrm{~V}, \mathrm{Vac}=0.1 \mathrm{mV}$

(a)
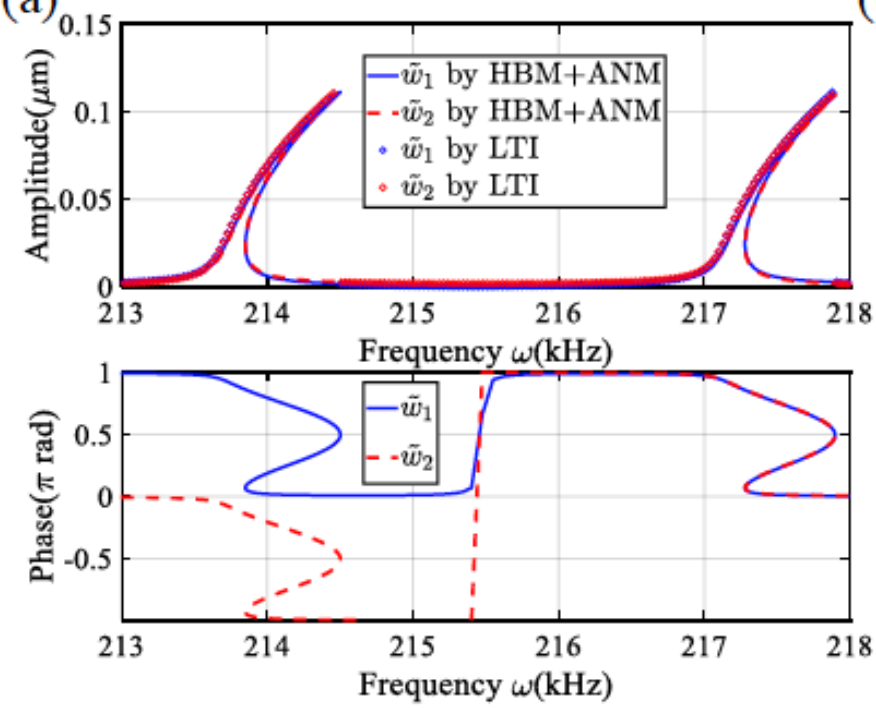

(b)
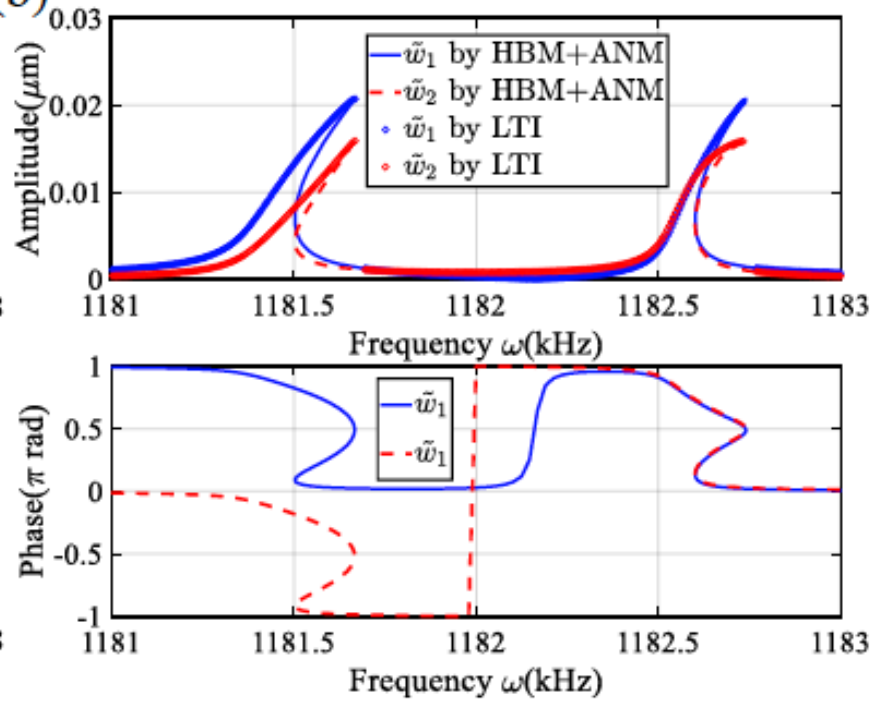

Figure 5

Nonlinear amplitude-frequency and phase-frequency curves of the two resonators with first order coupling (a) and third-second modes coupling (b) when $\mathrm{Vc}=10 \mathrm{~V}, \mathrm{Vac}=5 \mathrm{mV}$ 

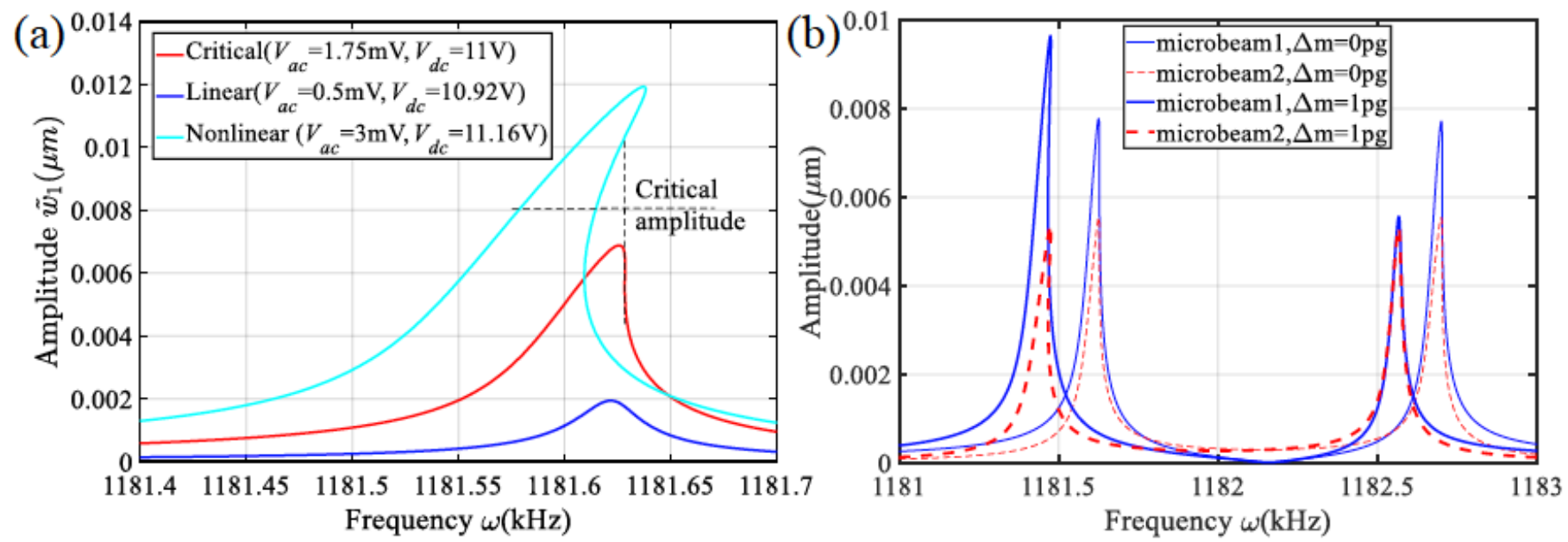

Figure 6

(a) amplitude of out-of-phase mode vibration of microbeam 1 with different Vac, (b) dynamic response of critical point with $\Delta \mathrm{m}=1 \mathrm{pg}$

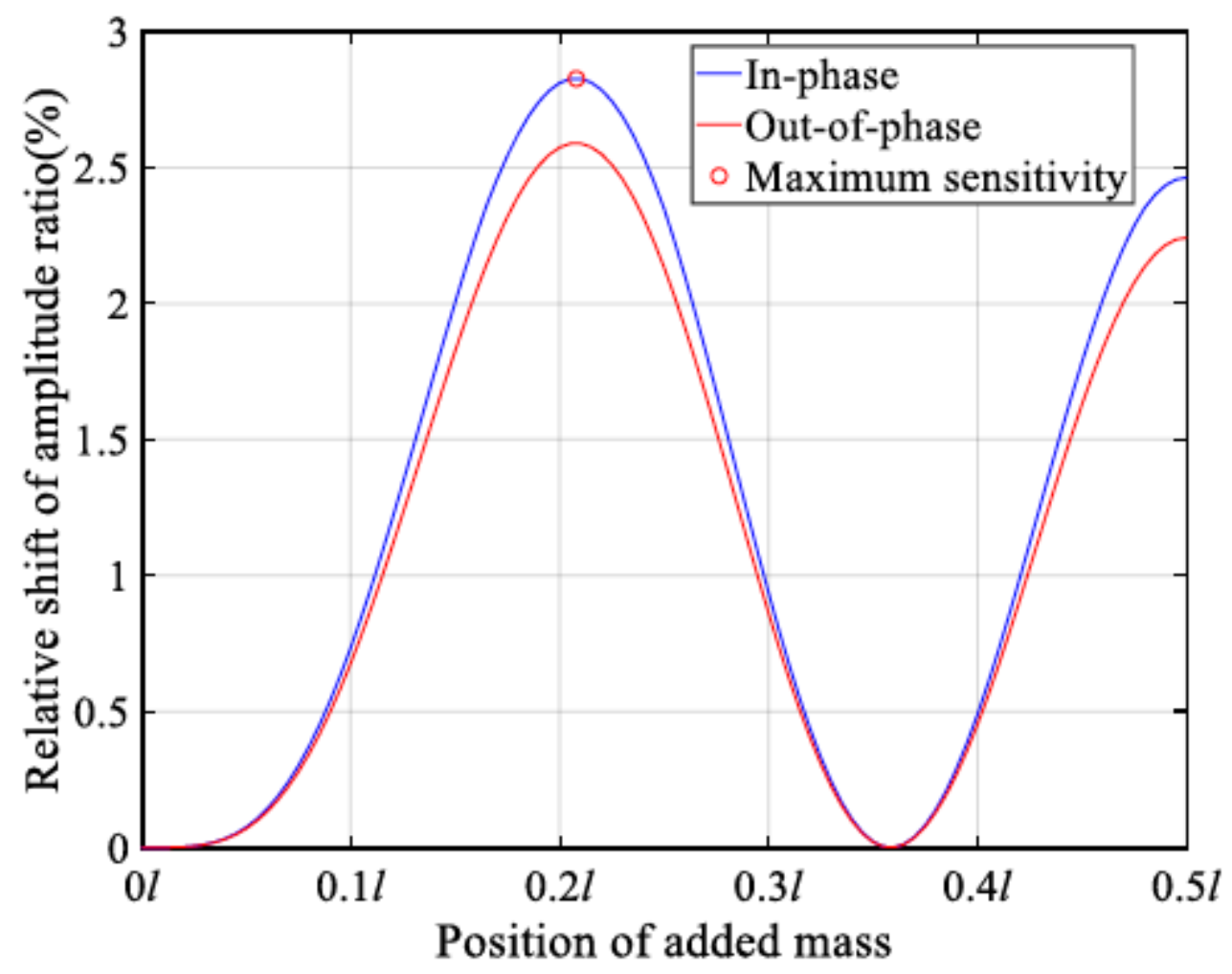

Figure 7

Influence of detection position on sensitivity with added mass of $0.1 \mathrm{pg}$ 
(a)

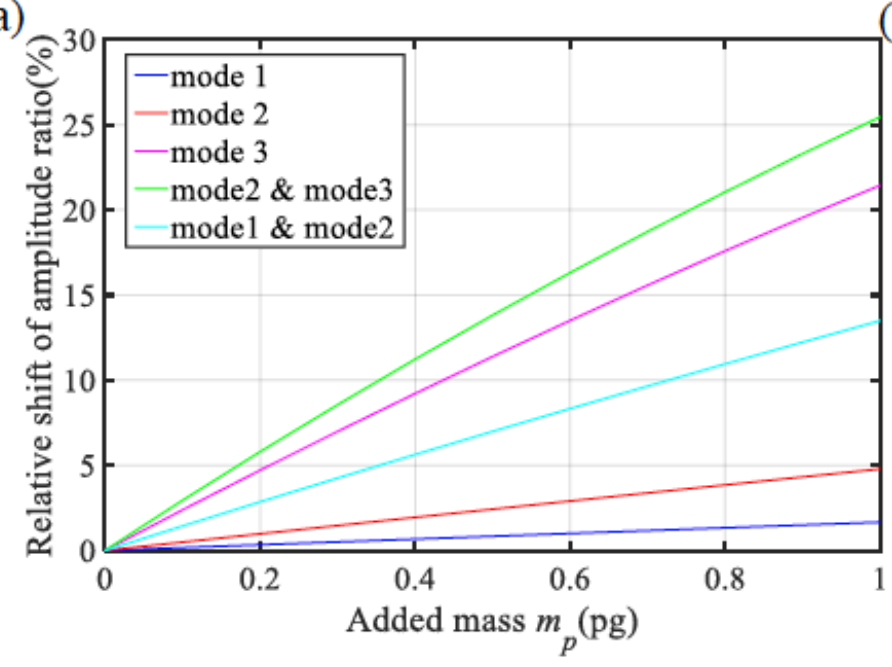

(b)

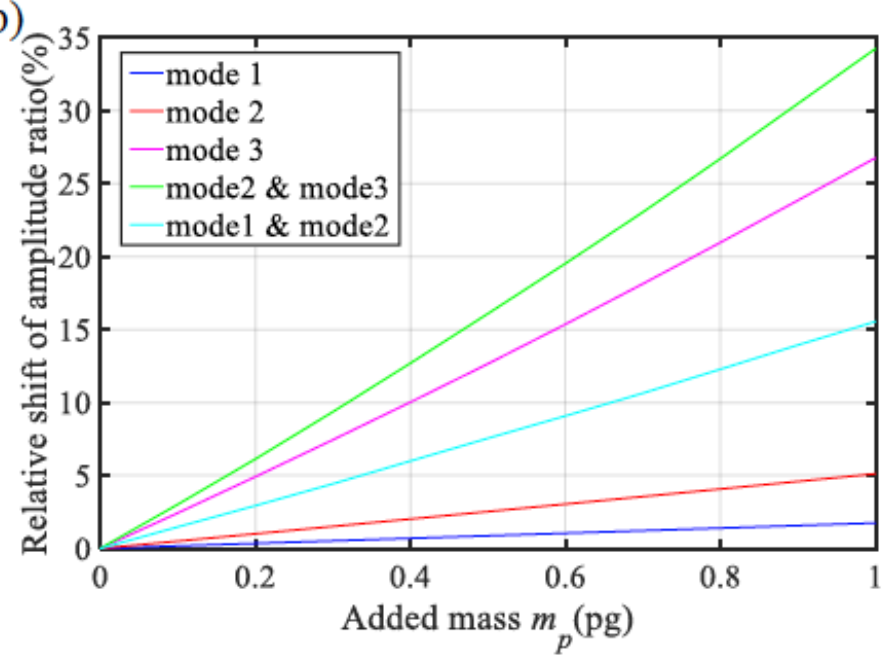

\section{Figure 8}

Sensitivity comparisons between sensors of the same order mode coupling and different order mode coupling when $\mathrm{Vac}=0.1 \mathrm{mV}$, out-of-phase mode $(a)$, in-phase mode (b) 

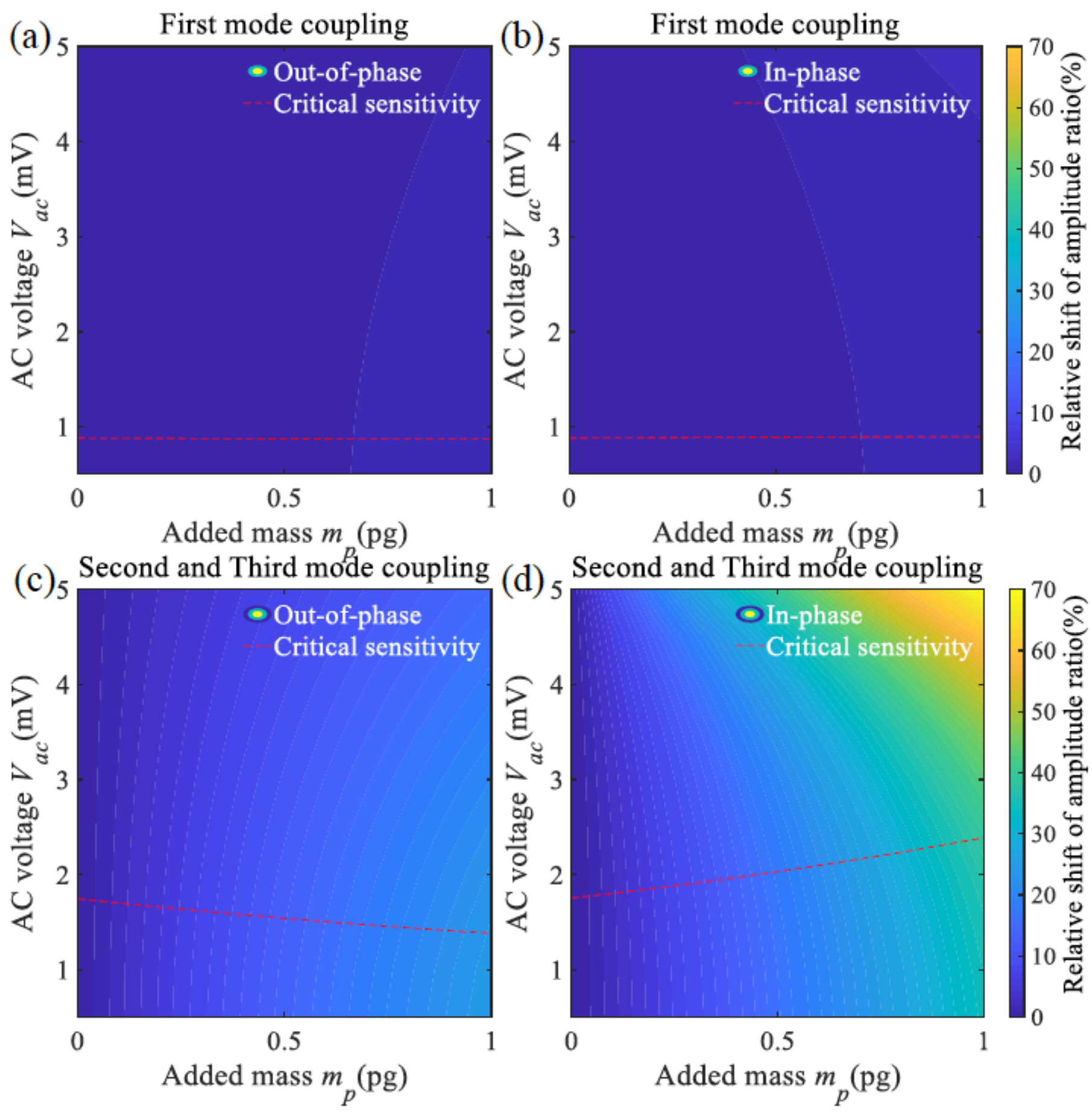

Figure 9

Relative shift of amplitude ratio with different AC voltage Vac, sensitivity of first mode coupling for out-ofphase (a) and in-phase mode (b), sensitivity of second and third mode coupling for out-of-phase (c) and in-phase mode $(d)$ 

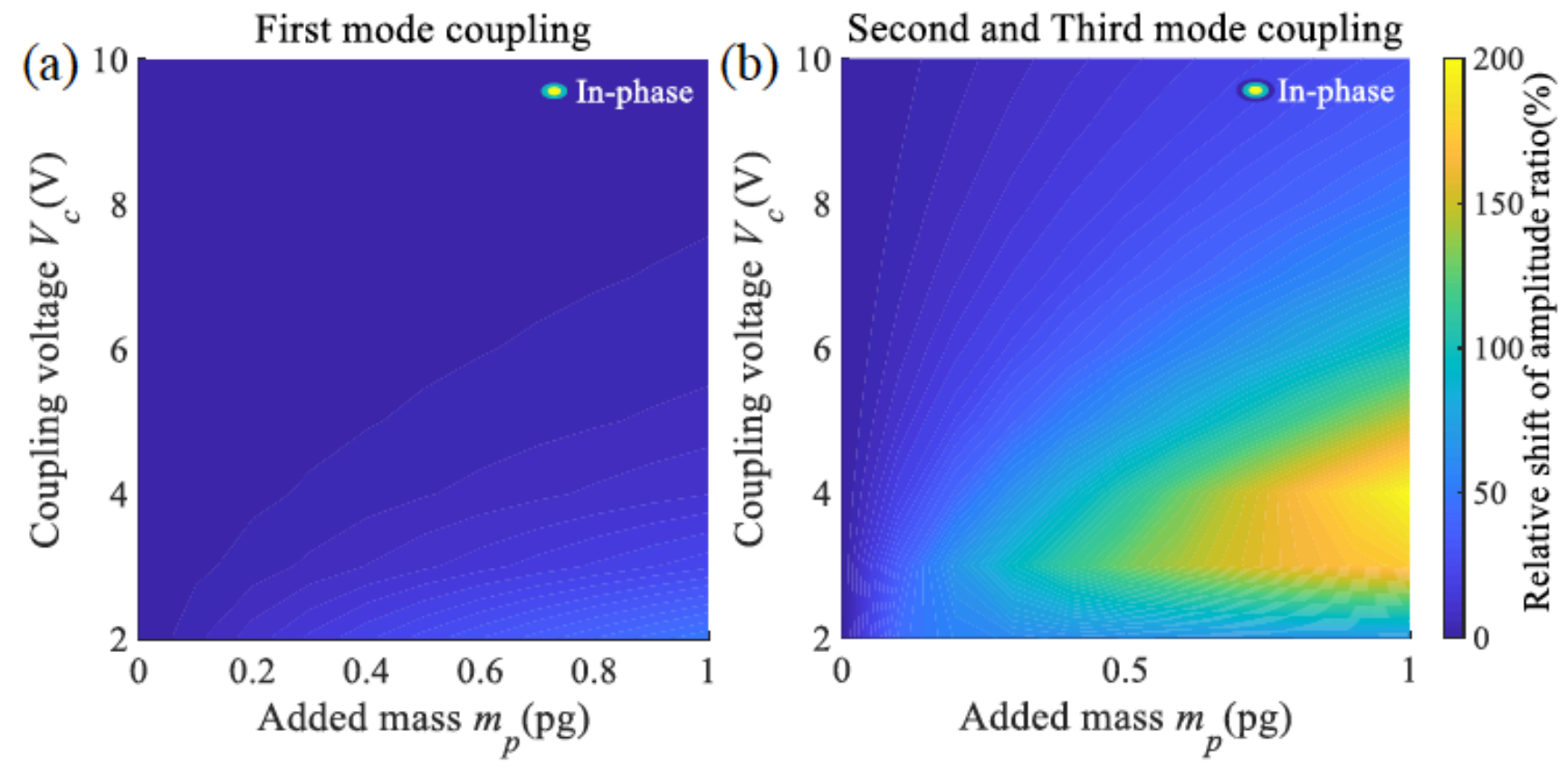

Figure 10

Relative shift of amplitude ratio with different coupling voltage $\mathrm{Vc}$, first mode coupling (a), second and third mode coupling (b) 